\title{
Volumen de la vía aérea superior en diferentes patrones esqueléticos faciales de una población peruana en tomografia computarizada de haz cónico
}

Upper airway volume in different facial skeletal patterns of a peruvian population in cone beam computed tomography.

\author{
Renzo Angel Paredes Vilchez ${ }^{1, a}$, Jaime Alejandro Hidalgo Chavez ${ }^{2, b}$
}

\section{RESUMEN}

Dentro del diagnóstico y plan de tratamiento en pacientes con deformidades dentofaciales la evaluación de la vía aérea superior es importante ya que ésta puede encontrarse alterada por el patrón esquelético facial o verse afectada por el tratamiento quirúrgico a realizar, siendo la Tomografía Computarizada de Haz Cónico (TCHC) el estudio de elección para medirla debido a su precisión y previsibilidad. Objetivo: Evaluar el volumen de la vía aérea superior en diferentes patrones esqueléticos faciales de una población peruana en TCHC. Materials y métodos :Se evaluaron 60 tomografias a través del programa PLANMECA Romexis Viewer, donde se midió el volumen en nasofaringe, orofaringe e hipofaringe según patrón esquelético facial y sexo. Resultados: El $45 \%$ perteneció al sexo masculino y el 55\% al femenino .Se observo que el volumen promedio más alto se encontró en los pacientes con Clase III con 7,37 $\mathrm{cm}^{3}, 19,14 \mathrm{~cm}^{3}$ y $5,65 \mathrm{~cm}^{3}$ en nasofaringe ,orofaringe e hipofaringe respectivamente .En hipofaringe se observó que en clase II y III los valores del volumen promedio son mayores en el sexo masculino significativamente $(\mathrm{P}<0.05)$.Además ,se encontró significancia de los patrones esqueléticos faciales en orofaringe e hipofaringe $(\mathrm{P}<0.05)$.Conclusiones: El volumen promedio de la vía aérea superior en pacientes con patrón esquelético facial Clase III es mayor que en Clase I y II , siendo significativo en orofaringe.

PALABRAS CLAVE: Sistema respiratorio, TCHC, Facies . 


\section{SUMMARY}

Within diagnosis and treatment planning in patients with dentofacial deformities, evaluation of the upper airway is important since it may be altered by facial skeletal pattern or be affected by orthognathic surgery, being the Cone Beam Computed Tomography (CBCT) the best diagnostic tool for evaluation of airway due to its precision and predictability. Objective: To evaluate upper airway volume in different facial skeletal patterns of a peruvian population in CBCT. Material and methods: 60 tomographies were evaluated through the PLANMECA Romexis Viewer program, where volume in nasopharynx, oropharynx and hypopharynx was measured. according to facial skeletal pattern and sex. Results: $45 \%$ were males and 55\% females. It was observed that the highest average volume was found in patients with Class III with $7.37 \mathrm{~cm}^{3}, 19.14 \mathrm{~cm}^{3}$ and $5.65 \mathrm{~cm}^{3}$ in nasopharynx, oropharynx and hypopharynx respectively. In hypopharynx it was observed that in class II and III the mean volume values are significantly higher in males $(\mathrm{P}<0.05)$. In addition, significance of facial skeletal patterns was found in oropharynx and hypopharynx $(\mathrm{P}<0.05)$. Conclusions: The average upper airway volume in patients with Class III facial skeletal pattern is greater than in Class I and II, being significant in oropharynx.

KEY WORDS: Respiratory system, CBCT, Facies.

\section{INTRODUCCIÓN}

Dentro del diagnóstico y plan de tratamiento en pacientes con deformidades dentofaciales el estudio de la vía aérea es importante ya que ésta puede encontrarse alterada por el patrón esqueletal o verse afectada por el tratamiento quirúrgico a realizar. Es así que encontramos estudios sobre la medida de la vía aérea superior por TCHC y su relación con el biotipo facial, patrón esquelético facial, sexo, edad entre otras variables (1-3). Guijarro-Martínez y Swennen (4), realizan y validan un estudio de medición de la vía aérea superior en TCHC, con limites anatómicos en nasofaringe, orofaringe e hipofaringe haciendo posible su reproducibilidad en otros estudios.La TCHC desde su aparición, ha sido de gran ayuda al campo de la cirugía bucal y maxilofacial debido a su fácil acceso, bajo costo y radiación comparada con la tomografía espiral multicorte (TEM). Es así que se ha convertido en una herramienta de elección para analizar la vía aérea tridimensionalmente (5).

La vía aérea superior es una estructura crítica en el sistema respiratorio humano. La morfología y sus dimensiones están regidas por estructuras anatómicas, como los tejidos blandos y el esqueleto craneofacial que rodea la faringe (6). El período de reposo para el desarrollo estructural faríngeo es más probable que se sitúe entre los 14 y los 18 años (7). Aunque Schendel SA et al (8) reportan el periodo de reposo hasta los 20 años, después del cual las vías respiratorias disminuyen lentamente de tamaño y que después de los 50 años ocurre más rápidamente.

En nuestro ámbito existen pocos estudios que miden la vía aérea superior basados en TCHC, sobre todo al tener una población variada como la peruana. El objetivo de este trabajo fue evaluar el volumen de la vía aérea superior en diferentes patrones esqueléticos faciales de una población peruana en TCHC.

\section{MATERIAL Y MÉTODOS}

Este estudio se realizó luego de obtener la aprobación del Instituto de Diagnóstico Maxilofacial (IDM) para la toma de muestra, así como de la Unidad Integrada de Gestión de Investigación , Ciencia y Tecnología(UIGCT) y aprobación del Comité Institucional de Ética en Investigación de la Universidad Peruana Cayetano Heredia (CIEI-UPCH) .El estudio fue de tipo transversal, retrospectivo, descriptivo y observacional.La población estuvo compuesta por las TCHC de pacientes que se realizaron el estudio en el IDM, sede Surco, en el periodo del 2017 al 2019.

Los criterios de inclusión requerían que los pacientes sean de origen peruano, dentición permanente y edad de 14 a 20 años. Los criterios de exclusión incluyeron pacientes con deformidades congénitas y adquiridas, antecedente de patología de vía aérea y antecedente de cirugía maxilofacial $u$ 


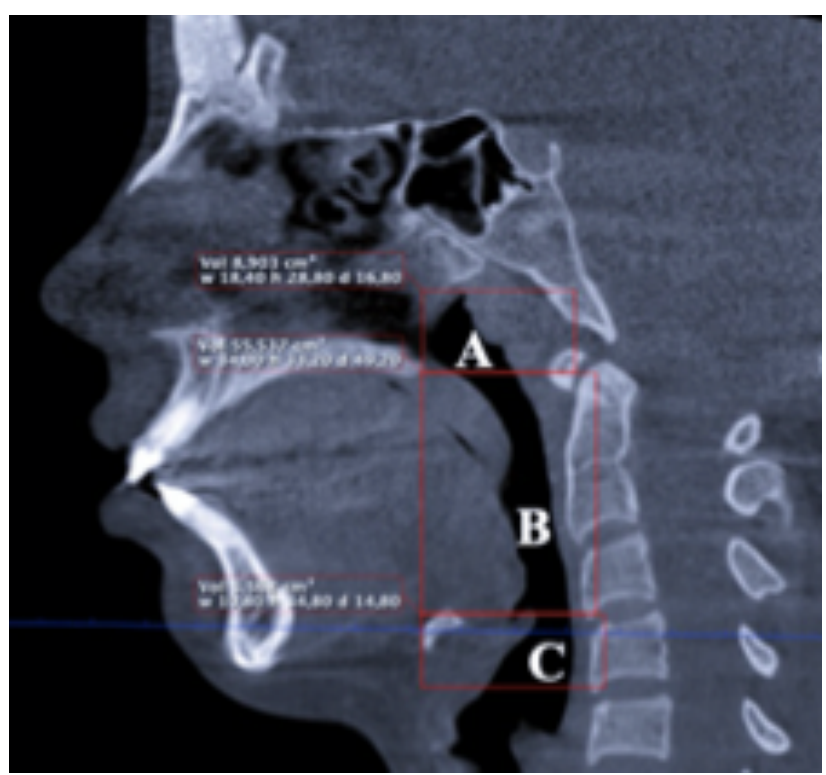

Figura1.Límites de la vía aérea superior(4): A. Nasofaringe; B. Orofaringe; C. Hipofaringe ortodoncia. La muestra del estudio estuvo compuesta por 60 pacientes $(20 \mathrm{TCHC}$ clase I, $20 \mathrm{TCHC}$ clase II, 20 TCHC clase III).Las TCHC fueron obtenidas de los archivos del equipo Planmeca Pro Max 3D Mid. El procedimiento de medición se realizó a través del software PLANMECA Romexis Viewer, a través del método de observación directa, en una pantalla de 21,5 pulgadas de la marca Apple iMac, donde se procedió a la ubicación de los limites anatómicos, según Guijarro-Martínez y Swennen (4).Donde la nasofaringe tiene su limite superior que va del contorno del tejido blando de la pared faríngea hasta su limite inferior en el plano paralelo a la Horizontal de Frankfort (HF) que pasa por la Espina Nasal Posterior (ENP) y se extiende a la pared posterior de la faringe. La orofaringe llega en su limite inferior al plano paralelo a $\mathrm{HF}$ que pasa por C3ai (punto mas anteroinferior del cuerpo de la tercera vertebra cervical) .La hipofaringe tiene su limite inferior en

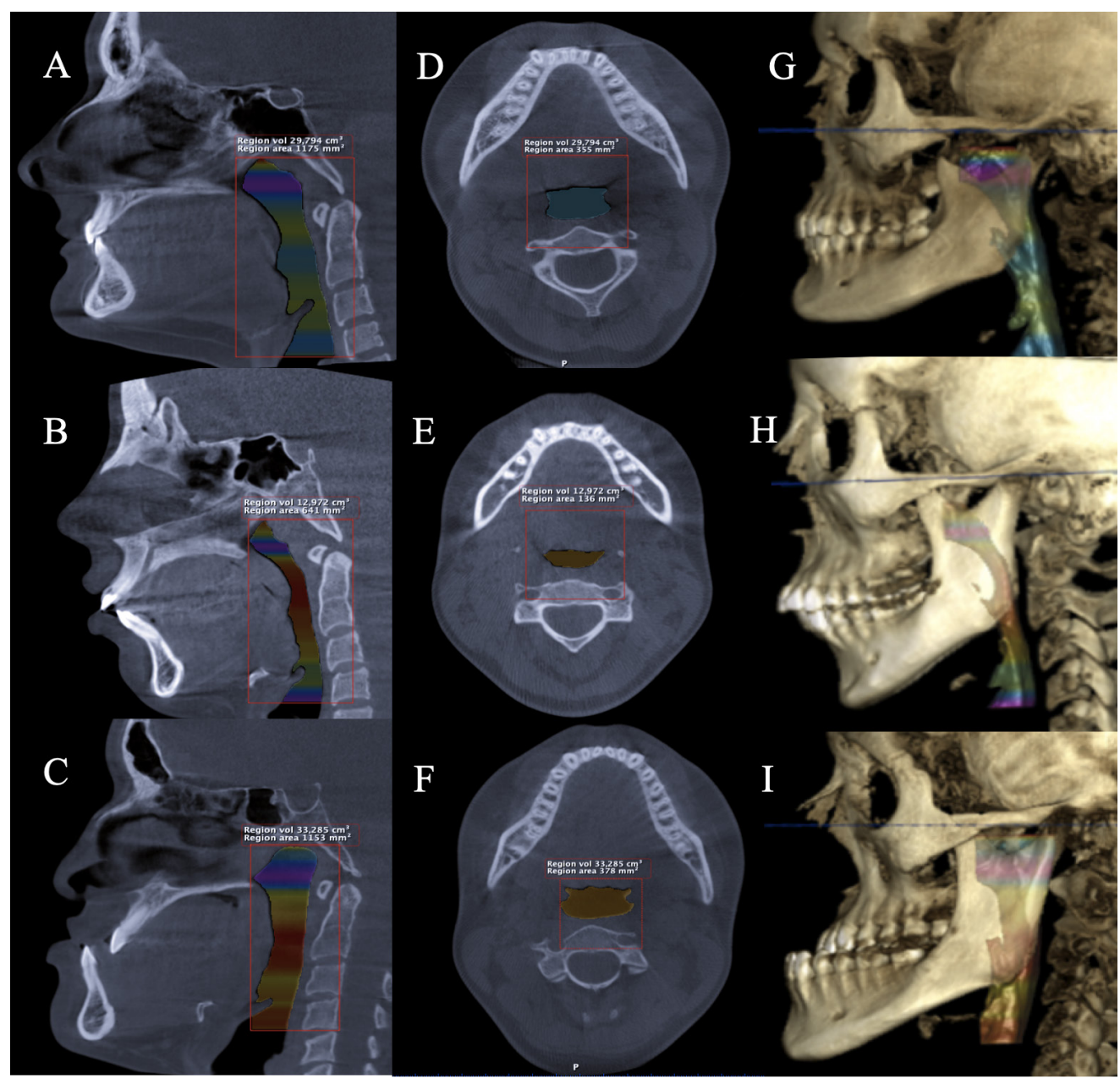

Figura 2. Vía aérea superior (Nasofaringe, Orofaringe e Hipofaringe). Clase I(A); Clase II(B) y Clase III (C). Vista axial a nivel de orofaringe en clase I (D); clase II (E) y clase III(F). Reconstrucción 3D del macizo facial y volumen de la vía aérea superior en pacientes clase I (G); II(H) y III(I). 
el plano paralelo a HF, conectando a la base de la Epiglotis o a la entrada del esófago (figura 1).

Se encontró el volumen en $\mathrm{cm}^{3}$ en cada patrón esquelético facial a través de la herramienta "región de crecimiento 3D", en cada segmento de la vía aérea superior, los cuales fueron clasificados según el ángulo ANB (Clase I: $1^{\circ} \leq \mathrm{ANB} \leq 3^{\circ}$, clase II: ANB $>3^{\circ}$, y clase III: $\mathrm{ANB}<1^{\circ}(7)$ )previamente a través de una vista sagital cefalométrica (figura 2).

Se trabajó los datos en el formato Excel y en Stata 16 , donde se diseñaron tablas para obtener la media, desviación estándar, valores mínimos y máximos. Se utilizó la prueba $t$ de Student para comparar dos medias independientes y la prueba $\mathrm{F}$ a través del ANOVA para comparar más de dos medias.

\section{RESULTADOS}

Delas 60TCHC evaluadas, 27 (45\%) pertenecieron al sexo masculino y 33 (55\%) al femenino.

En los pacientes Clase I se observó que el volumen promedio más alto se encontró en orofaringe con $15,27 \mathrm{~cm}^{3}$ y una desviación estándar de $5,77 \mathrm{y}$ el volumen promedio más bajo en hipofaringe con 4.48 $\mathrm{cm}^{3}$ y una desviación estándar de 1,12 (tabla 1).

Con respecto a los pacientes Clase II se encontró que el volumen promedio más alto se presentó en orofaringe con 13,08 $\mathrm{cm}^{3}$ y una desviación estándar de 4,04 y el volumen promedio más bajo en la hipofaringe con $4,57 \mathrm{~cm}^{3}$ y una desviación estándar de 1,42 (tabla 2).

En los pacientes Clase III se obtuvo que el volumen promedio más alto se presentó en orofaringe con $19,14 \mathrm{~cm}^{3}$ y una desviación estándar de 4,59 y el volumen promedio más bajo en la hipofaringe con $5,65 \mathrm{~cm}^{3}$ y una desviación estándar de 2,16 (tabla 3).

Tabla 1. Volumen de la vía aérea superior en Patrón Esquelético Facial I.

\begin{tabular}{llllll}
\hline Variable & $\mathbf{n}^{\circ}$ & Media & D.S. & Min & Max \\
\hline Nasofaringe & 20 & 5,92 & 2,19 & 2,3 & 11 \\
Orofaringe & 20 & 15,27 & 5,77 & 8,7 & 29 \\
Hipofaringe & 20 & 4,48 & 1,12 & 2,6 & 7.2 \\
\hline
\end{tabular}

Tabla 2. Volumen de la vía aérea superior en Patrón Esquelético Facial II.

\begin{tabular}{llllll}
\hline Variable & $\mathbf{n}^{\circ}$ & Media & D.S. & Min & Max \\
\hline Nasofaringe & 20 & 6,47 & 1,65 & 4,4 & 10,1 \\
Orofaringe & 20 & 13,08 & 4,04 & 5,9 & 19,6 \\
Hipofaringe & 20 & 4,57 & 1,42 & 2,4 & 7,2 \\
\hline
\end{tabular}

Tabla 3. Volumen de la vía aérea superior en Patrón Esquelético Facial III.

\begin{tabular}{llllll}
\hline Variable & $\mathbf{n}^{\circ}$ & Media & D.S. & Min & Max \\
\hline Nasofaringe & 20 & 7,37 & 2,33 & 4,1 & 15,1 \\
Orofaringe & 20 & 19,14 & 4,59 & 11,1 & 26,8 \\
Hipofaringe & 20 & 5,65 & 2,16 & 2,6 & 10,1 \\
\hline
\end{tabular}

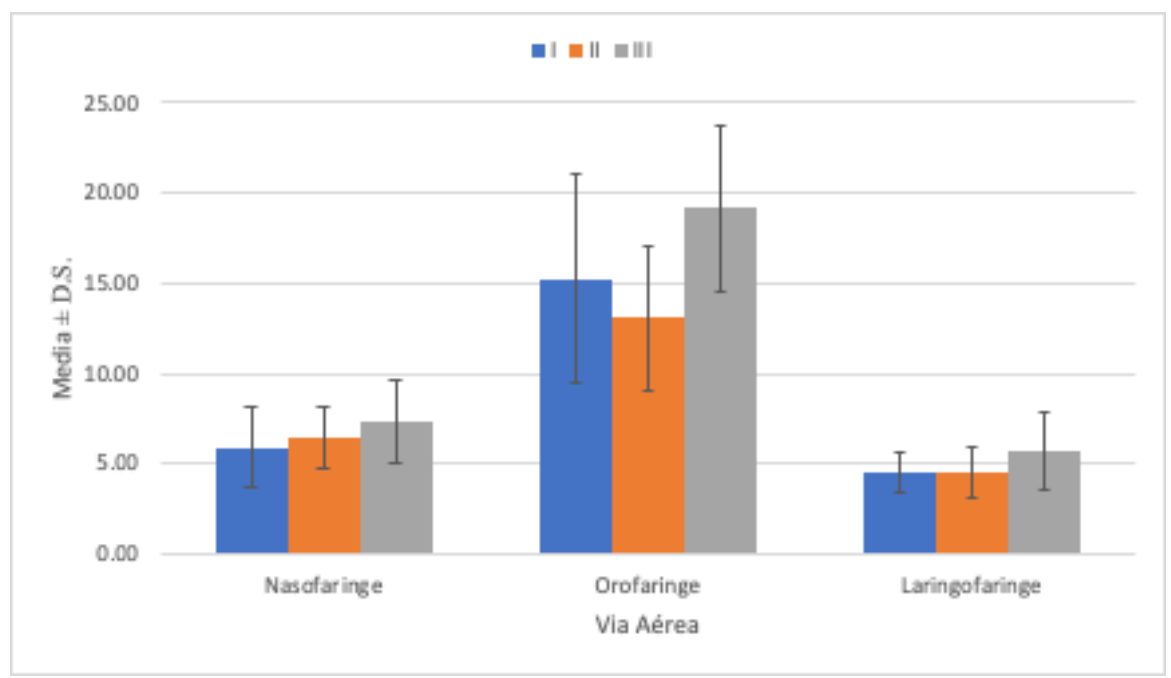

Gráfico 1. Volumen de la vía aérea superior en Patrón Esquelético I, II y III. 
Al evaluar la vía aérea superior en los patrones esqueléticos según sexo se observó en la nasofaringe, un promedio mayor en mujeres dentro de la clase I $(6,667 \pm 2,19$ en mujeres y $4,788 \pm 1,747$ en hombres), mientras que en clase II y clase III los valores promedio son similares entre hombres y mujeres. En orofaringe, el volumen promedio es mayor en mujeres en comparación a los hombres en la clase I con un promedio de 17,19 $\pm 6,36$. En clase II el promedio es ligeramente mayor en los hombres con un promedio de $13,525 \pm 4,109$ y en mujeres un promedio de $12,783 \pm 4,146$, en clase III es mayor en hombres con un promedio de 19,682 $\pm 3,356$.

En hipofaringe, el volumen promedio es ligeramente mayor en hombres dentro de la clase I $(4,863 \pm 1,537$ en hombres y 4,217 $\pm 0,683$ en mujeres), en clase II y clase III los valores del volumen promedio son mayores en el sexo masculino y esas diferencias son estadísticamente significativas, $\mathrm{P}<0,05$ (tabla 4).

Tabla 4. Volumen de la vía aérea superior en diferentes Patrones Esqueléticos Faciales según sexo.

\begin{tabular}{|c|c|c|c|c|c|c|c|c|c|}
\hline \multirow[b]{2}{*}{ Sexo } & \multicolumn{3}{|c|}{ Clase I } & \multicolumn{3}{|c|}{ ClaseII } & \multicolumn{3}{|c|}{ ClaseIII } \\
\hline & $\mathbf{n}$ & Media & D.S. & $\mathbf{n}$ & Media & D.S. & $\mathbf{n}$ & Media & D.S. \\
\hline Nasofaringe $F$ & 12 & 6,667 & 2,193 & 12 & 6,417 & 1,541 & 9 & 7,111 & 3,243 \\
\hline \multirow[t]{2}{*}{ M } & 8 & 4,788 & 1,747 & 8 & 6,538 & 1,919 & 11 & 7,582 & 1,353 \\
\hline & \multicolumn{3}{|c|}{$\mathrm{t}=2,027 \quad \mathrm{P}=0,0578$} & \multicolumn{3}{|c|}{$t=-0,156 P=0,878$} & \multicolumn{3}{|c|}{$\mathrm{t}=-0,439 \mathrm{P}=0,66$} \\
\hline \multicolumn{10}{|l|}{ Orofaringe } \\
\hline $\mathrm{F}$ & 12 & 17,192 & 6,36 & 12 & 12,783 & 4,146 & 9 & 18,467 & 5,921 \\
\hline \multirow[t]{2}{*}{ M } & 8 & 12,375 & 3,278 & 8 & 13,525 & 4,109 & 11 & 19,682 & 3,356 \\
\hline & \multicolumn{3}{|c|}{$\mathrm{t}=1,963 \quad \mathrm{P}=0,065$} & \multicolumn{3}{|c|}{$t=-0,393 P=0,69$} & \multicolumn{3}{|c|}{$\mathrm{t}=-0,57 \mathrm{P}=0,570$} \\
\hline \multicolumn{10}{|l|}{ Hipofaringe } \\
\hline $\mathrm{F}$ & 12 & 4,217 & 0,683 & 12 & 4,017 & 1,062 & 9 & 4,189 & 1,64 \\
\hline \multirow[t]{2}{*}{ M } & 8 & 4,863 & 1,537 & 8 & 5,388 & 1,562 & 11 & 6,845 & 1,786 \\
\hline & \multicolumn{3}{|c|}{$\mathrm{t}=-1,29 \mathrm{P}=0,214$} & \multicolumn{3}{|c|}{$\mathrm{t}=-2,34 \mathrm{P}=0,03^{*}$} & \multicolumn{3}{|c|}{$\mathrm{t}=-3,43 \mathrm{P}=0,003^{*}$} \\
\hline
\end{tabular}

t: Prueba t de Student, P: Nivel de significancia, ${ }^{*}$ diferencia significativa

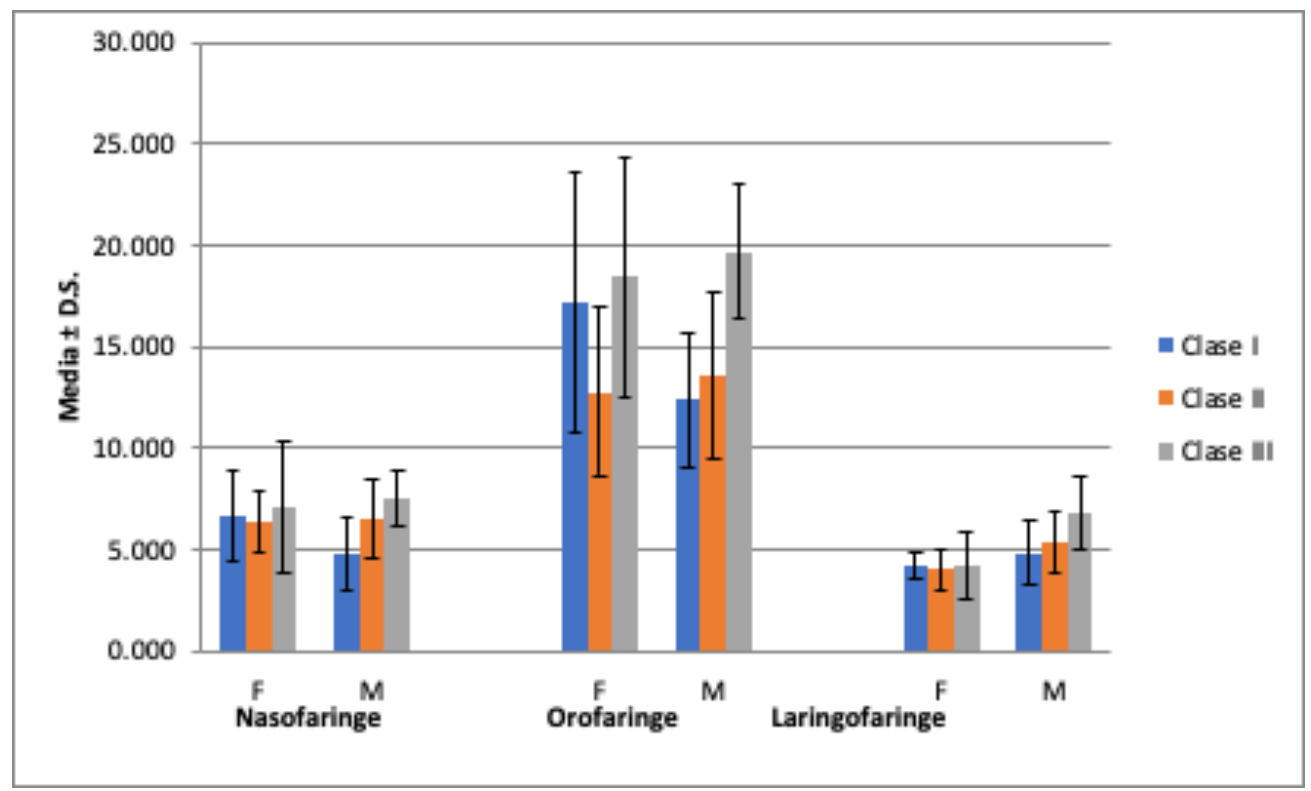

Gráfico 2. Volumen de la vía aérea superior en diferentes Patrones Esqueléticos según sexo. 
Tabla 5. Volumen de la vía aérea superior según diferentes Patrones Esqueléticos Faciales.

\begin{tabular}{lcccccccc}
\hline Vía Aérea Superior & $\begin{array}{c}\text { I } \\
\text { Media }\end{array}$ & D.S. & $\begin{array}{c}\text { II } \\
\text { Media }\end{array}$ & D.S. & Media & D.S. & & \\
\hline Nasofaringe & 5,92 & 2,19 & 6,47 & 1,65 & 7,37 & 2,33 & 2,49 & 0,0916 \\
Orofaringe * & 15,27 & 5,77 & 13,08 & 4,04 & 19,14 & 4,59 & 7,99 & 0,0009 \\
Hipofaringe** & 4,48 & 1,12 & 4,57 & 1,42 & 5,65 & 2,16 & 3,24 & 0,0465 \\
\hline $\begin{array}{l}{ }^{*} \text { significativo entre I y III, II y III } \\
* * \text { significativo entre I y III }\end{array}$ & & & & & & &
\end{tabular}

Al evaluar la vía aérea superior según patrón esquelético, el volumen promedio en nasofaringe más alto se encontró en los pacientes con Clase III $(7,37$ $\left.\mathrm{cm}^{3}\right)$ seguido por los de Clase II $\left(6,47 \mathrm{~cm}^{3}\right)$ y Clase I $\left(5,92 \mathrm{~cm}^{3}\right)$; en orofaringe fue mayor en los pacientes Clase III $\left(19,14 \mathrm{~cm}^{3}\right)$, seguido por los de Clase I $\left(15,27 \mathrm{~cm}^{3}\right)$ y Clase II $\left(13,08 \mathrm{~cm}^{3}\right)$.En hipofaringe el volumen promedio fue superior en los de Clase III $\left(5,65 \mathrm{~cm}^{3}\right)$, seguido por los de Clase $\mathrm{II}\left(4,57 \mathrm{~cm}^{3}\right)$ y Clase I $\left(4,48 \mathrm{~cm}^{3}\right)$. Con respecto a la relación entre los grupos se encontró que fue significativa en orofaringe e hipofaringe $(\mathrm{P}<0,05)$ (tabla 5).

\section{DISCUSIÓN}

El estudio de la vía aérea superior en diferentes patrones esqueléticos faciales es importante ,ya que puede ser alterado por la cirugía ortognática. Siendo la TCHC la mejor herramienta de diagnóstico para su evaluación tridimensional. En la literatura existen pocos estudios de la evaluación de la vía aérea en poblaciones mestizas como la peruana, por lo que el objetivo de este trabajo fue evaluar el volumen de la vía aérea superior en diferentes patrones esqueléticos faciales de una población peruana en TCHC.

Se evaluaron 60 tomografías, siendo la mayoría del sexo femenino coincidiendo con el estudio de Zheng et al., y Castro-Silva et al., (9); esto podría deberse a que la población femenina busca en mayor proporción tratamientos estéticos. Según Dos Santos et al., la población femenina busca corrección de la oclusión, con mejoras en la estética facial (10).

El volumen promedio nasofaríngeo encontrado en pacientes Clase I, II y III fue $5,9 \mathrm{~cm}^{3}, 6.4 \mathrm{~cm}^{3}$ y $7.3 \mathrm{~cm}^{3}$ respectivamente; Zheng et al encontraron valores parecidos de $5,4 \mathrm{~cm}^{3}, 4,05 \mathrm{~cm}^{3}$ y 6,05 $\mathrm{cm}^{3}$. Claudino et al., (2) describieron el volumen orofaringe e hipofaringe como porción faríngea inferior, con valores en pacientes Clase I, II y III de $20,5 \mathrm{~cm}^{3}, 21,1 \mathrm{~cm}^{3}$ y $29,9 \mathrm{~cm}^{3}$, respectivamente .El presente estudio tuvo como media $19,7 \mathrm{~cm}^{3}, 17,6 \mathrm{~cm}^{3}$ y $25 \mathrm{~cm}^{3}$. Las diferencias encontradas pueden deberse al origen étnico de las poblaciones estudiadas , así como por la cantidad de muestra evaluada. Estos estudios tuvieron como mayor volumen promedio de vía aérea superior a los pacientes de Clase III. Esto puede deberse a un mayor volumen de la vía aérea en orofaringe e hipofaringe por la posición anterior de la mandibula con respecto a la maxila. Según Kikuchi esto es debido a que la vía aérea está influenciada por la posición anteroposterior del hueso mandibular (11). Observando que el diámetro interno de la vía aérea inferior se expande anteriormente cuando el hueso mandibular esta en posición anterior y que se contrae levemente y se alarga verticalmene cuando la mandibula rota posteriormente.

El estudio de la vía aérea superior según sexo no mostró diferencias significativas en nasofaringe tal como en el estudio de Zheng et al, sin embargo, se encontraron valores promedios superiores en hipofaringe en individuos Clase II y III del sexo masculino siendo estadísticamente significativas $(\mathrm{P}<0,05)$. Esto puede deberse al dimorfismo sexual que encontramos en la porción laríngea. Según Mohsein hay mayor limitación de la vía aérea superior en mujeres debido a la anatomía local del cartílago cricoides (12).

Castro-Silva et al encontraron que el promedio de la vía aérea en clase III fue mayor que en I y II. Así como Iwasaki et al., quien refiere que la clase III se asocia con un espacio orofaríngeo mayor que la Clase I (13). El presente estudio encontró que los volúmenes 
en los individuos de clase III son mayores que las otras clases en nasofaringe, orofaringe e hipofaringe, siendo significativo en la última $(\mathrm{P}<0,05)$. Esto puede estar explicado por el estudio de Hong et al., quienes observaron que la vía aérea faríngea tenía correlación con la posición anterior mandibular (14). De forma contraria, mandíbulas colocadas posteriormente puede llevar la lengua y el paladar blando a reducir el volumen de la vía aérea superior (7).

Huamaní encontró que sujetos con patrón dolicofacial, registraron menor volumen en orofaringe que los mesofaciales y braquifaciales, pero no fue estadísticamente significativo (1). Por lo que podríamos decir que la medida de la vía aérea superior puede variar con el biotipo facial, patrón esquelético facial, sexo, edad entre otras variables. Aunque la significancia de estos valores está asociada al tamaño de la muestra y la distribución por edades según Zheng et al.; esto se podría explicar a los amplios rangos de la vía aérea en sus valores mínimos y máximos.

La medición y valoración de la vía aérea superior aporta información valiosa durante el diagnóstico y la planificación quirúrgica .En el meta-análisis realizado por He et al., se evidencia que la cirugía bimaxilar promueve una menor disminución en la vía aérea superior que otras cirugías para la corrección de la maloclusión tipo III (15). Así como Wiedemeyer et al., obtuvieron la predicción del aumento del volumen de la vía aérea faríngea después del avance mandibular en pacientes Clase II (16). De igual manera autores como Cheng-Hui Lin (17) y Faur (18) muestran que el avance bimaxilar y rotación antihoraria maxilomandibular son técnicas empleadas en pacientes con presencia de volumen de la vía aérea disminuida, con resultados estables y disminución de los índices de apnea.

La valoración de la vía aérea superior en planificación para cirugía ortognática debe ser contrastada con una TCHC en el preoperatorio y otra en el postoperatorio, el cual es una limitación de este estudio, al ser de tipo retrospectivo. Por lo cual se recomienda que futuras investigaciones tomen medidas postoperatorias de la vía aérea superior, con controles tomográficos mayores a 6 meses para controlar la estabilidad de la vía aérea y de la cirugía.

\section{CONCLUSIONES}

Los valores volumétricos en nasofaringe, orofaringe e hipofaringe en individuos con diferente patrón esquelético de una población peruana son muy variables con resultados máximos que fueron hasta 4 veces mayor que los valores mínimos.

Se encontró que el volumen de la vía aérea superior en diferentes patrones esqueléticos según sexo, son mayores en el sexo masculino en hipofaringe con diferencias significativas para los pacientes clase II y III. Además, se encontró relación entre el volumen de la vía aérea superior según patrón esquelético , siendo estadísticamente significativo en orofaringe, donde fue mayor la clase III ,seguido por la clase I y II respectivamente.

Agradecimientos : Al Instituto de Diagnóstico Maxilofacial (IDM) y a los Drs. Andrés Marco Agurto Huerta y Eduardo Miguel Calle Velezmoro, por facilitarnos el uso de sus equipos ,así como por la capacitación del uso del software para la realización del presente trabajo de investigación.

\section{Correspondencia:}

Renzo Angel Paredes Vilchez

Correo electrónico: renzo.paredes@upch.pe

\section{REFERENCIAS BIBLIOGRÁFICAS}

1. Huamani H. Volumen de la vía orofaríngea según el biotipo facial en tomografías cone beam de pacientes que acudieron al Instituto de Diagnóstico Maxilofacial. Tesis para optar el Título Profesional de Cirujano Dentista. Lima: Universidad Nacional Mayor de San Marcos; 2016.

2. Claudino LV, Mattos CT, de Oliveira Ruellas AC, Sant'Anna EF. Pharyngeal airway characterization in adolescents related to facial skeletal pattern: a preliminary study. Am J Orthod Dentofacial Orthop. 2013;143(6):799- 809 .

3. Zheng Z, Yamaguchi T, Kurihara A, Li H, Maki K. Three-dimensional evaluation of upper airway in patients with different anteroposterior skeletal patterns. Orthod Craniofac Res. 2014;17(1):38-48.

4. Guijarro-Martínez R, Swennen GR. Three dimensional cone beam computed tomography definition of the anatomical subregions of the upper airway: a validation study. Int J Oral Maxillofac Surg. 2013; 42:1140-9.

5. Guijarro-Martínez R, Swennen GR. Cone-beam 
computerized tomography imaging and analysis of the upper airway: a systematic review of the literature. Int J Oral Maxillofac Surg. 2011;40(11):1227-37.

6. Shokri A, Miresmaeili A, Ahmadi A, Amini P, FalahKooshki S. Comparison of pharyngeal airway volume in different skeletal facial patterns using cone beam computed tomography. J Clin Exp Dent. 2018;10(10): E1017-e28.

7. El H, Palomo JM. Airway volume for different dentofacial skeletal patterns. Am J Orthod Dentofacial Orthop. 2011; 139:511-21.

8. Schendel SA, Jacobson R, Khalessi S. Airway growth and develop- ment: a computerized 3-dimensional analysis. J Oral Maxillofac Surg. 2012; 70:2174-83.

9. Castro-Silva L, Monnazzi MS, Spin-Neto R, et al. Cone-beam evaluation of pharyngeal airway space in class I, II, and III patients. Oral Surg Oral Med Oral Pathol Oral Radiol. 2015;120(6):679-83.

10. Dos Santos LF, Albright DA, Dutra V, Bhamidipalli SS, Stewart KT, Polido WD. Is there a correlation between airway volume and maximum constriction area location in different dentofacial deformities? J Oral Maxillofac Surg. 2020;78(8):1415.e1-1415.e10.

11. Kikuchi Y. Three-dimensional relationship between pharyngeal airway and maxillo-facial morphology. Bull Tokyo Dent Coll. 2008;49(2):65-75.

12. Mohsenin V. Gender differences in the expression of sleep-disordered breathing: role of upper airway dimensions. Chest. 2001;120(5):1442-7

13. Iwasaki T, Hayasaki H, Takemoto $Y$, Kanomi R, Yamasaki Y. Oropharyngeal airway in children with Class III malocclusion evaluated by cone-beam computed tomography. Am J Orthod Dentofacial Orthop. 2009;136(3): 318.e1-319.

14. Hong JS, Oh KM, Kim BR, Kim YJ, Park YH. Threedimensional analysis of pharyngeal airway volume in adults with anterior position of the mandible. Am J Orthod Dentofacial Orthop. 2011;140(4): e161-e169.

15. He J, Wang Y, Hu H, et al. Impact on the upper airway space of different types of orthognathic surgery for the correction of skeletal class III malocclusion: A systematic review and meta-analysis. Int J Surg. 2017; 38:31-40.

16. Wiedemeyer V, Berger M, Martini M, Kramer FJ, Heim N. Predictability of pharyngeal airway space dimension changes after orthognathic surgery in class II patients: A mathematical approach. J Craniomaxillofac Surg. 2019;47(10):1504-9.

17. Cheng-Hui C, Wang PF, Ray Han-Loh S, Lau HT, ShengPing S. Maxillomandibular Rotational Advancement: Airway, Aesthetics, and Angle's Considerations. Sleep Med Clin. 2019;14(1):83-9.

18. Faur CI, Roman RA, Bran S, et al. The Changes in Upper Airway Volume after Orthognathic Surgery Evaluated by Individual Segmentation on CBCT Images.Maedica.2019;14(3):213-19.

Recibido : 14-12-2020 Aceptado : 15-06-2021 